\title{
Anomalous left pulmonary artery without pulmonary artery sling
}

\author{
A Robida, B Venkatraman
}

An anomalous left pulmonary artery almost always runs posterior to the trachea causing a pulmonary artery sling compressing the trachea and oesophagus. We describe a unique case of an anomalous left pulmonary artery passing anterior to the trachea without producing the pulmonary artery sling associated with a right aortic arch and an aberrant left subclavian artery.

\section{Case report}

A 5 year old asymptomatic girl presented with a heart murmur. Echocardiography established a clinical diagnosis of a large secundum atrial septal defect. A right aortic arch was also noted. The origin of the left pulmonary artery

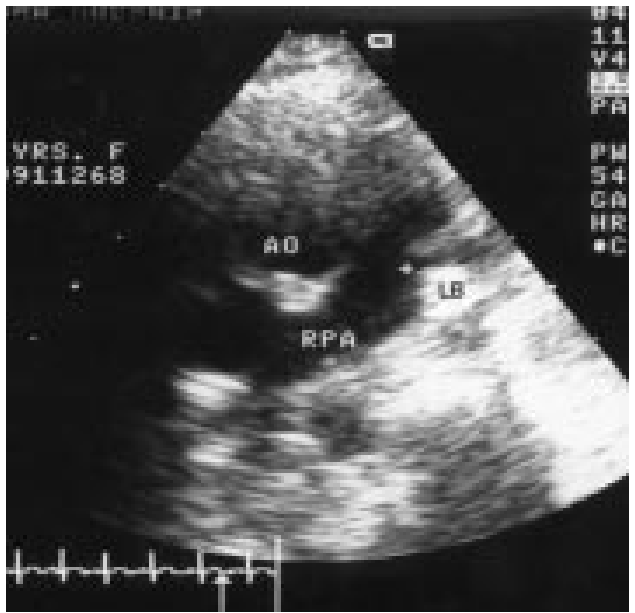

Figure 1 A modified suprasternal view of the right pulmonary artery (RPA) with an anomalous origin of the left pulmonary artery (arrow) anterior to the left main bronchus (LB). AO, aorta.

Cardiology and

Cardiovascular

Surgery, Division of

Paediatric Cardiology,

Hamad General

Hospital, PO Box 3050 ,

Doha Qatar

A Robida

Department of

Radiology, Hamad

General Hospital

B Venkatraman

Correspondence to: Dr Robida.

Accepted for publication 7 January 1998
Figure 2 A posteroanterior angiogram into the pulmonary trunk showing the origin of the left pulmonary artery (arrow) from the right pulmonary artery just at the level of the tracheal bifurcation. was not seen from the pulmonary trunk and was subsequently seen to arise from the right pulmonary artery anterior to the trachea (fig 1). Oesophagography showed an impression on the right upper lateral margin by the right aortic arch with an additional posterior oblique indentation, consistent with an aberrant left subclavian artery. There was no anterior oesophageal indentation. The anomalous origin of the left pulmonary artery was confirmed by angiography (fig 2 ) and by surgical inspection during closure of the atrial septal defect.

\section{Discussion}

A pulmonary artery sling, a rare anomaly, is formed when the left pulmonary artery originates anomalously from the right pulmonary artery and passes between the trachea and the oesophagus. ${ }^{1}$ Embryogenesis of this anomaly is based on the evaluation of the reported cases. Variants of this anomaly have recently been described emphasising the developmental hypothesis of Jue and associates. ${ }^{2-4}$ A case report of a partial anomalous left pulmonary artery has been published where the branch of the left

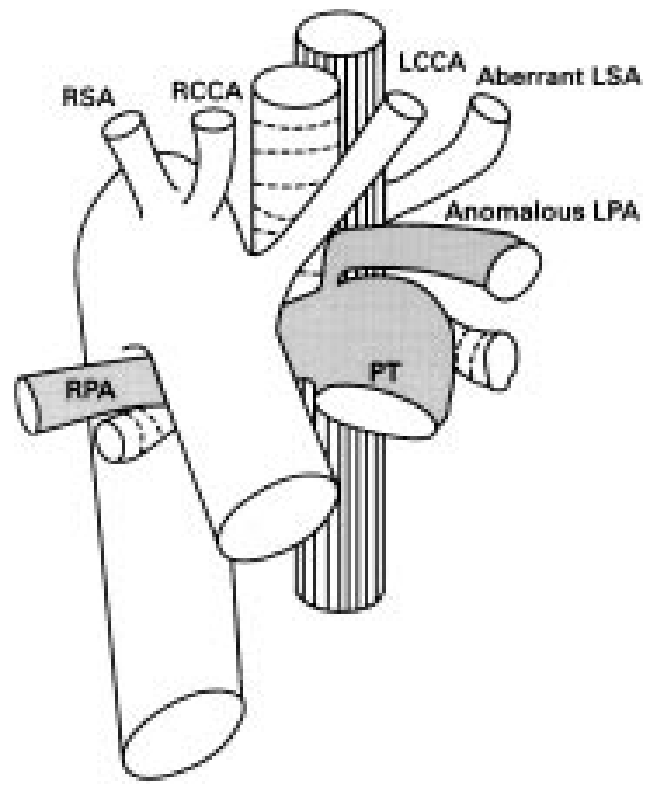

Figure 3 Drawing of the anomaly showing the anomalous left pulmonary artery in relation to adjacent structures. LCCA, left common carotid artery; LPA, left pulmonary artery; LSA, left subclavian artery; PT, pulmonary trunk; RCCA, right common carotid artery; RPA, right pulmonary artery; $R S A$, right subclavian artery. 
pulmonary artery supplying the left lower lung lobe passed behind the trachea forming a sling. The branch for the left upper lung lobe originated in a normal fashion from the main pulmonary artery. ${ }^{3}$ In another case of a partially anomalous left pulmonary artery the arrangement of the left pulmonary artery was similar except that the branch of the left lower lobe pulmonary artery ran in front of the trachea producing no pulmonary artery sling. ${ }^{4}$

The distal parts of the pulmonary arteries normally develop from the capillaries of their respective lung buds. These are then joined with the ipsilateral sixth aortic arches. ${ }^{5}$ The embryology of the anomalous left pulmonary artery passing dorsally to the trachea has been described as a failure of the left lung buds' connection with the left sixth arch. Instead, the connection of the left lung buds occurs with the right sixth arch dorsally to the trachea forming the pulmonary artery sling. ${ }^{2}$ If this connection runs ventrally no sling is formed. The presence of the right aortic arch and the aberrant left subclavian artery in our patient is important because this anomaly of the aortic arch is believed to be a consequence of the involution of the left forth aortic arch and loss of the left sixth aortic arch. Because of the loss of the left sixth aortic arch it is plausible that the left lung buds could not connect to it. A connection with the right sixth aortic arch has thus formed creating the anomalous left pulmonary artery (fig 3). We think that the involution of the fourth left aortic arch and loss of the left sixth aortic arch, if occurring before or at the same time as the faulty connection of the left pulmonary artery, could explain the coexistence of these anomalies.

The two cases with the partially anomalous left pulmonary artery, one running anteriorly and the other posteriorly to the trachea, and our patient with the completely anomalous left pulmonary artery passing in front of the trachea in combination with the right aortic arch and the aberrant left subclavian artery, lend strong support for the developmental hypothesis of Jue and associates. ${ }^{2}$

1 Welsh TM, Munro IB. Congenital stridor caused by an aberrant pulmonary artery. Arch Dis Child 1954;29:101-4.

2 Jue KL, Raghib G, Amplatz, et al. Anomalous origin of the left pulmonary artery from the right pulmonary artery: report of 2 cases and review of the literature. Am F Roentgenol Radium Ther Nucl Med 1965;95:598-610.

3 Bamman JL, Ward BH, Woodroom DE. Aberrant left pulmonary artery. Chest 1977;72:67-71.

pulmonary artery. Chest 1977;72:67-71.
4 Erickson LC, Cocalis MW, George L. Partial anomalous left Erickson LC, Cocalis MW, George L. Partial anomalous left
pulmonary artery: new evidence on the development of the pulmonary artery sling. Pediatr Cardiol 1996;17:319-21.

5 Huntington GS. The morphology of the pulmonary artery in the mammalia. Anat Rec 1919;17:165-90. 